\title{
Autonomic mediation of unconditioned and conditioned heart rate responses in the 16-day-old rat
}

\author{
PAMELA S. HUNT, MAUREEN F. HESS, and BYRON A. CAMPBELL \\ Princeton University, Princeton, New Jersey
}

\begin{abstract}
In three experiments, we examined the cardiac response of 16-day-old rats to a 10-sec olfactory stimulus before and after aversive Pavlovian conditioning. The unlearned (orienting) response to the novel stimulus was bradycardia, and the conditioned response was a brief period of bradycardia early in the conditioned stimulus, followed by pronounced tachycardia. After selective blockade of the parasympathetic and sympathetic systems with atropine or atenolol, the deceleratory orienting response was found to be the result of an increase in vagal discharge, accompanied by a cooccurring decrease in sympathetic stimulation of the heart. The initial deceleratory component of the conditioned response was produced by an increase in parasympathetic stimulation of the heart, and the acceleratory component was generated by an increase in sympathetic activity. These results are discussed in terms of their significance for the neural and autonomic origins of the phasic changes in heart rate that are elicited by the olfactory stimulus during different stages of conditioning.
\end{abstract}

Conditioned and unconditioned changes in heart rate (HR) have been widely used as indices of learning and attention (Campbell, Hayne, \& Richardson, 1992; Coles, Jennings, \& Stern, 1984; Graham \& Clifton, 1966; Kimmel, van Olst, \& Orlebeke, 1979; Lacey \& Lacey, 1970). In light of the wide use of HR as an indicator of functional/adaptive reactions, it is especially critical to understand the physiological origin of these response patterns. Complexities in the autonomic determinants of changes in HR arise from recent challenges to the traditional assumption that the sympathetic (SNS) and parasympathetic (PNS) systems serve reciprocal functions. It is now widely recognized that changes in the activity of both divisions may be seen independently and even concurrently (D. H. Cohen \& Pitts, 1968; Gold \& D. H. Cohen, 1981; Iwata \& LeDoux, 1988; Quigley \& Berntson, 1990; Richardson, Wang, \& Campbell, 1994). In view of this, a change in HR may be relatively uninformative regarding its underlying autonomic control. For example, an increase in HR may result from activation of the SNS, withdrawal of the PNS, or reciprocal activation and inhibition of the SNS and PNS, respectively.

The present studies were designed to investigate the autonomic origins of the conditioned increase in HR that occurs when an olfactory stimulus is paired with shock

This research was supported by National Institutes of Child Health and Human Services postdoctoral Grant HD07694 to P.S.H. and National Institute of Mental Health Grants MH01562 and MH49496 to B.A.C. The authors wish to thank Rick Richardson and Matthew Kurtz for many helpful discussions and comments on earlier versions of the paper. Requests for reprints should be addressed to B. A. Campbell, Department of Psychology, Green Hall, Princeton University, Princeton, NJ 08544. in the preweanling rat (Sananes, Gaddy, \& Campbell, 1988). Little is known about the integration of central neural control of conditioned phasic changes in HR in response to sensory stimuli during development (Hofer \& Reiser, 1969; Larson \& Porges, 1982; Saiers, Richardson, \& Campbell, 1989), and determining the autonomic origins of conditioned tachycardia in the preweanling rat is of interest for several reasons.

First, neither the sympathetic nor the parasympathetic divisions of the autonomic nervous system is fully mature at that age (Adolph, 1965, 1968; H. L. Cohen, 1986; Hofer, 1974; Larson \& Porges, 1982; Slotkin, 1986; Tucker \& Domino, 1988). The two divisions develop asynchronously and somewhat idiosyncratically. For example, application of acetylcholine to the heart decreases HR earlier in development than application of catecholamines increases HR in many species, including the rat (Adolph, 1965, 1968). Similarly, direct stimulation of the vagus decreases HR earlier in development than direct stimulation of the sympathetic nerves increases HR (Adolph, 1968; Pappano, 1977). Paradoxically, autonomic control of basal HR appears to develop in the reverse order. Tonic SNS stimulation of the heart appears to emerge some time during the first week postnatally (Adolph, 1965; Larson \& Porges, 1982; Tucker, 1985), although Slotkin argues that this occurs somewhat later, during the second postnatal week. In contrast, there is little or no evidence suggesting that tonic PNS control of basal HR emerges until the beginning of the third week of life (Adolph, 1968; Hofer \& Reiser, 1969; Tucker, 1985).

Second, there is increasing evidence that the autonomic mechanisms mediating phasic cardiac responses to some stressor stimuli are different in the infant rat 
than in the adult. Haroutunian and Campbell (1982), for example, reported that the tachycardia elicited by footshock in 19-day-old rats resulted from PNS withdrawal rather than from activation of the SNS as it is in the adult (Katcher et al., 1969; Kazis, Milligan, \& Powell, 1973). Similarly, Kurtz and Campbell (in press) found that a moderately intense white-noise stimulus elicited tachycardia in periadolescent and adult rats, but that the increase was mediated by PNS withdrawal in the periadolescents and reciprocal PNS withdrawal and SNS activation in the adults. Furthermore, recent research from our laboratory has shown that an intense acoustic startle stimulus $(130 \mathrm{~dB})$ produces a PNS-mediated 25-30 beats-per-minute (BPM) decrease in HR in the preweanling rat and a 15-30 BPM SNS-driven increase in the adult (Richardson et al., 1994). Collectively, these reports reveal that there are numerous instances in which the same environmental stressor stimulus elicits different patterns of autonomic activity during the course of ontogeny. As Schneirla $(1959,1965)$ has postulated, the PNS in its control over phasic responses is more primitive than the SNS, and it may subserve functions that are particular to the early postnatal niche of mammals (Hofer \& Reiser, 1969).

The purpose of the present experiments was to determine whether the conditioned increase in HR to an olfactory CS paired with a shock unconditioned stimulus (US) in preweanling rats is mediated through an increase in SNS activation, PNS withdrawal, or concurrent changes in SNS and PNS activation of the heart. The aim of Experiment 1 was to confirm the conditioned increase in HR to an olfactory stimulus reported by Sananes et al. (1988) before proceeding to the autonomic analysis. In the following two experiments, we examined the autonomic division(s) mediating the unconditioned decrease in HR elicited by the novel olfactory stimulus characteristic of the orienting response (Experiment 2) and the conditioned acceleratory response to the olfactory conditioned stimulus (CS; Experiment 3).

\section{EXPERIMENT 1}

The purpose of Experiment 1 was to replicate the conditioned increase in HR to an olfactory stimulus reported by Sananes et al. (1988). A second purpose was to analyze the changes in HR elicited by the CS on a second-by-second basis in order to determine whether the unconditioned and conditioned responses were monophasic, or had more complex forms.

\section{Method}

Subjects. The subjects were 20 experimentally naive 16-dayold Sprague-Dawley rats from seven litters, born in the psychology department's vivarium at Princeton University. On Postnatal Day 2 (day of birth, Day 0), the litters were culled to 8 animals. The animals were raised with the dam and littermates in standard $48.0 \times 25.5 \times 20.5 \mathrm{~cm}$ polycarbonate maternity cages with wood chips as bedding. Purina Lab Chow and water were available ad lib. The colony was maintained on a 16:8-h light:dark illumination cycle, with light onset at $0700 \mathrm{~h}$. All testing was conducted during the light portion of the cycle. No more than 2 animals from each litter served in a given experimental condition. All procedures employed were approved by the Princeton University Animal Care and Use Committee.

Apparatus. The conditioning chamber was a Plexiglas cylinder, $14 \mathrm{~cm}$ in diameter and $25 \mathrm{~cm}$ long, mounted horizontally in a custom built, sound-attenuating chamber with interior dimensions measuring $66 \times 37 \times 81.5 \mathrm{~cm}$. A 7.5 -W white bulb was mounted on an inside wall of this chamber to provide constant low-level illumination. The olfactory stimulus $(0.25 \mathrm{ml}$ amyl acetate diluted in $40 \mathrm{ml}$ water) was introduced into the cylinder by way of an airstream (main stream) that was generated by an air pump (Thomas) kept at $30^{\circ} \mathrm{C}$ by passing it through a water-heated coil. Another smaller airstream, generated from the same air pump, passed either through a flask containing water to produce a "neutral" airstream, or through a flask containing the liquid odorant. The airstream was controlled by two T-valves, one before and one after the two flasks, and joined the main stream shortly before entering the Plexiglas cylinder. The neutral airstream remained on at all times, except during the 10 -sec olfactory stimulus presentations. During this time, the valves were switched, shunting the airstream to the flask containing the odorant. The odor was evacuated from the cylinder by negative pressure generated by an exhaust fan. The US was produced by a constant-current shock generator. Shock was delivered through the HR recording electrodes.

Heart rate was recorded through two transcutaneous steel electrodes, shaped like small safety pins, with attached teflon-coated leads (32 ga; Alpha Wire). Electrodes were acutely implanted-one ventrally near the urogenital region and the other dorsally at the nape of the neck. Implantation required approximately $10-15 \mathrm{sec}$; the electrodes and leads did not appear to interfere with the animals' movements or general comfort. Cardiac potentials obtained through these electrodes were amplified by a Grass preamplifier (Model P15). The R-spike activated a Schmitt trigger (Coulbourn Instruments). A microcomputer stored each interbeat interval (IBI) to the nearest millisecond. The computer controlled all timing sequences and data collection. The cardiac signal was continuously displayed on a Hitachi oscilloscope (Model V-212).

Procedure. The animals were removed from the home cage and randomly assigned to one of two treatment groups $(n s=10)$, designated according to whether they received paired or explicitly unpaired presentations of the olfactory CS and shock US. Electrodes were implanted, and an animal was placed into the chamber for a 15 -min period of adaptation. The onset of the first trial was delayed if the subject was grooming (see Richardson, Hess, \& Campbell, 1994; Richardson, Siegel, \& Campbell, 1988).

Paired animals were given 15 pairings of the 10 -sec olfactory $\mathrm{CS}$, terminating with the onset of a 1-sec $0.6-\mathrm{mA}$ transcutaneous shock US. A variable intertrial interval (range, 75-285 sec) separated each pairing. Unpaired subjects also received $15 \mathrm{CS}$ (interstimulus intervals ranged from 75 to $285 \mathrm{sec}$ ) and 15 USs. These were presented in an explicitly unpaired and alternating manner, with the stipulation that a US could not occur $30 \mathrm{sec}$ before or after a CS.

Statistical analyses. Interbeat intervals were measured during a 5-sec prestimulus period and during the 10 -sec stimulus period on each trial. The IBI data were converted to a BPM measure for analysis. The mean HR during the last second of the prestimulus baseline was subtracted from the mean HR during each of the $10 \mathrm{sec}$ of the stimulus, resulting in a difference score for each second of the CS. Negative difference scores indicated cardiac deceleration (bradycardia) during the CS, and positive difference scores indicated cardiac acceleration (tachycardia).

The change from baseline HR on each of the $10 \mathrm{sec}$ of the CS were averaged across blocks of three trials, yielding a total of five trial blocks. The data were analyzed by using 2 (conditioning treatment) $\times 10$ (seconds) mixed analyses of variance (ANOVAs) for each trial block. Where appropriate, post hoc comparisons 
were made by using Neuman-Keuls tests $(p=.05)$. In all cases involving a repeated measure, the Greenhouse-Geisser method was used to control for potential inflation in the actual probability values (Keppel, 1982).

\section{Results}

Acquisition of the conditioned HR response. The acquisition data for paired and unpaired subjects are presented in Figure 1. In this figure, the data are expressed as the second-by-second change from baseline HR, collapsed across blocks of three training trials. Panels A-C depict Trial Blocks 1, 3, and 5, respectively. For the first trial block, the ANOVA yielded a significant main effect of seconds $[F(9,162)=12.04, p<.01]$.

For both paired and unpaired groups, a significant reduction in HR was observed. This was followed by a gradual return toward baseline over the $10 \mathrm{sec}$ of the CS. The magnitude of the deceleration did not differ significantly between the two groups (Figure 1A).

The analyses conducted on Trial Blocks 2-5 all yielded significant main effects of conditioning treatment [smallest $F(1,18)=5.94, p<.05$ ] and seconds [smallest $F(9,162)=12.40, p<.01]$, and significant conditioning treatment $\times$ seconds interactions [smallest $F(9,162)=$ $6.62, p<.01]$. Beginning with the second block of three trials, the paired and unpaired animals expressed different patterns of HR changes over the 10 -sec CSs. The paired group began to exhibit conditioned tachycardia on Block 2. Contrast analyses confirmed that the tachycardia seen during the latter part of the CS was significant relative to the paired group's prestimulus baseline on each of Trial Blocks 2-5 [smallest $F(10,90)=14.07$, $p<.01]$. The initial, small-magnitude bradycardia ob- served for the first few seconds after stimulus onset approached, but did not attain, statistical significance. In contrast, the unpaired group displayed habituation of the initial deceleratory response, and the change from baseline HR was a small and generally nonsignificant bradycardia throughout the remaining training trials. Contrast analyses indicated that this bradycardia was significantly different from the unpaired group's prestimulus baseline for 2-3 sec on each of Trial Blocks 2-4 [smallest $F(1,9)=3.21, p<.05]$.

Baseline HR. A 2 (conditioning treatment) $\times 5$ (trial block) mixed ANOVA was conducted on the prestimulus baseline HRs. This analysis yielded a main effect of trial block $[F(4,72)=10.17, p<.01]$. The main effect of conditioning treatment and the interaction were both nonsignificant. Baselines decreased slightly throughout the conditioning procedure, and basal HRs of the paired and unpaired subjects did not differ on any trial block.

\section{Discussion}

These results document the transition from an unconditioned deceleratory to a conditioned acceleratory cardiac response in preweanling rats during pairings of an olfactory CS with a shock US, confirming the findings of Sananes et al. (1988). The animals in the paired condition initially expressed unconditioned bradycardia to the novel stimulus, but with continued training exhibited a reliable tachycardia. This conditioned tachycardia was evident by the second block of three training trials and was significant with respect to this group's prestimulus baseline HR as well as to the response of the explicitly unpaired control subjects. Moreover, the conditioned response tended to be delayed with respect to stimulus

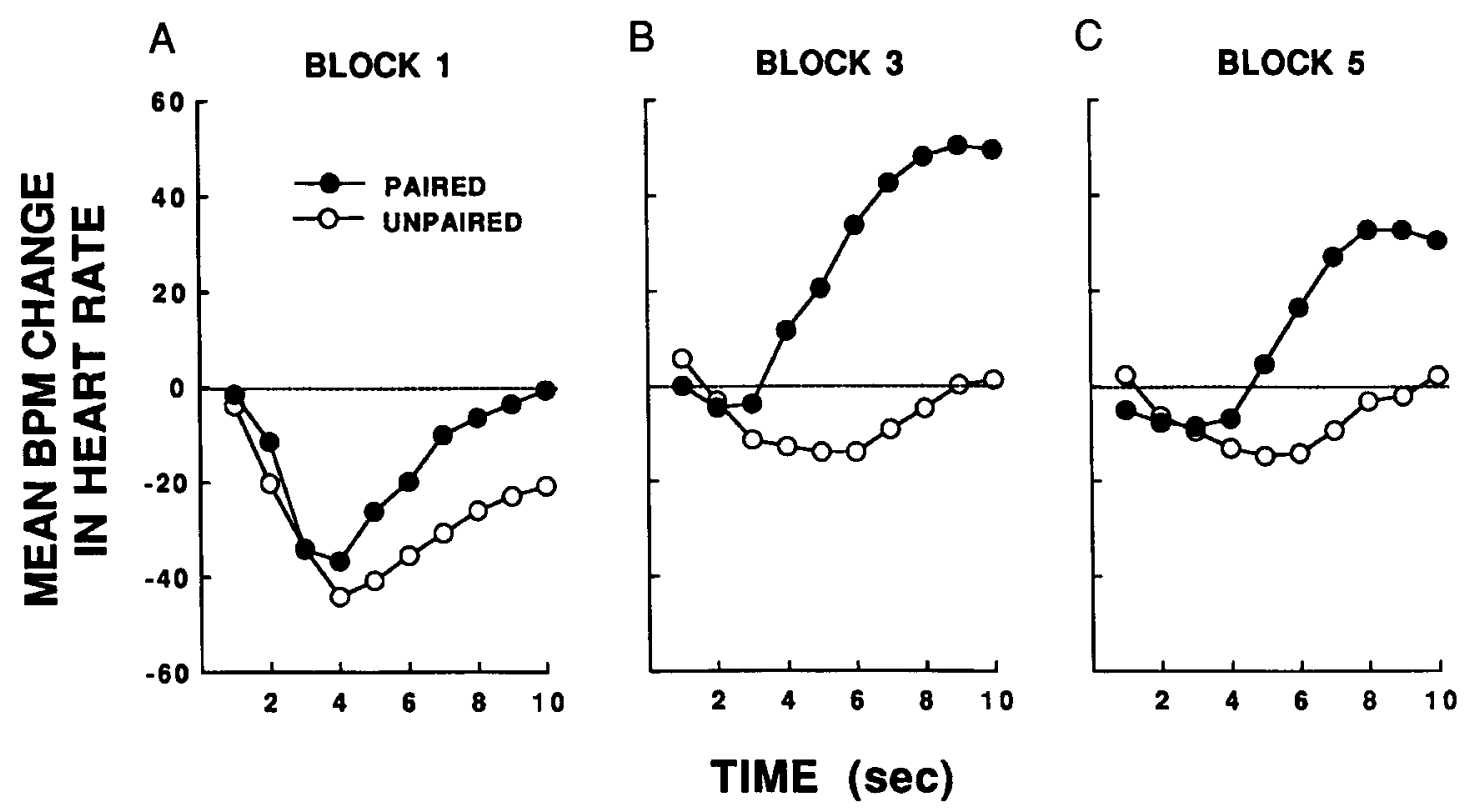

Figure 1. Mean beats-per-minute (BPM) change in heart rate to an olfactory stimulus after either paired or explicitly unpaired presentations of the odor conditioned stimulus with transcutaneous shock. Panels $\mathrm{A}-\mathrm{C}$ depict the heart rate changes for Trial Blocks 1 (Trials 1-3), 3 (Trials 7-9), and 5 (Trials 13-15), respectively, of Experiment 1. 
onset, with the acceleratory component's not emerging until approximately the 5th or 6th sec.

It has been suggested that changes in HR reflect changes in somatomotor behavior (Obrist, 1968; Obrist, Wood, \& Perez-Reyes, 1965). A fearful animal may exhibit a wide variety of responses, including either freezing or flight (Fanselow, 1991), and changes in HR may covary with motoric demands placed upon the organism, either in preparation for or execution of these motor patterns. Previous work by Sananes et al. (1988, Experiment 3 ), however, suggests that the degree of cardioacceleration seen during the olfactory CS was not correlated with the amount of activity concurrently recorded. This indicates that the HR CR is not a byproduct of CS-elicited changes in somatomotor responding.

\section{EXPERIMENT 2}

Experiment 2 was designed to examine the autonomic origins of the unconditioned HR response to the novel olfactory stimulus used in Experiment 1 . Unconditioned HR responses to the odor CS were evaluated after selective blockade of either the sympathetic or parasympathetic division by using atenolol as the $\beta$-adrenergic blocking agent and atropine methyl nitrate as the muscarinic cholinergic antagonist. According to previous reports, activation of the PNS mediates this orienting response (Dykman \& Gantt, 1959; Graham, 1979; Lacey \& Lacey, 1970; Saiers et al., 1989).

\section{Method}

Subjects. The subjects were 70 experimentally naive 16 -day-old $( \pm 1)$ rats from 14 litters, maintained as those in Experiment 1 . No more than 1 animal per litter was assigned to a given treatment group.

Apparatus. The apparatus was the same as that described in Experiment 1. Additionally, atenolol and atropine methyl nitrate (Sigma) were diluted in physiological saline to achieve doses of $0.2,0.4$, and $0.8 \mathrm{mg} / \mathrm{kg}$. The saline vehicle was injected at a volume that was equal to the largest $(0.8 \mathrm{mg} / \mathrm{kg})$ dose of each agent.

Procedure. The animals were removed from the home cage, injected intraperitoneally with one of the three doses of atropine or atenolol, or the saline vehicle ( $n s=10$ /group), and HR recording electrodes were acutely implanted. The animals were then placed into the Plexiglas chamber, maintained at $30^{\circ} \mathrm{C}$, for a 15 -min period of adaptation. After this time, five $10-\mathrm{sec}$ olfactory stimuli were presented on a variable interstimulus interval (range, $90-120 \mathrm{sec}$ ). The first trial was delayed if a subject was grooming. Heart rate was recorded during a $5-\mathrm{sec}$ prestimulus period and during the 10 -sec olfactory stimulus, as described in Experiment 1 . The change from baseline HR was determined for each second of the 10-sec CS.

\section{Results}

Preliminary analyses of the data obtained from the saline group with a 5 (trial) $\times 10$ (seconds) mixed ANOVA yielded a significant main effect of trials $[F(4,144)=16.14, p<.01]$, indicating the occurrence of habituation of the unconditioned HR response to the olfactory CS. Post hoc comparisons verified that the response to the olfactory stimulus in this group was significantly smaller on Trials 4 and 5 than on Trial 1 . Therefore, the data were collapsed across the first three trials for purposes of further analysis and illustration.

Atropine. As can be seen in Figure 2A, administration of atropine prior to odor presentations attenuated, but did not completely eliminate, the unconditioned HR deceleration observed in the saline-pretreated group. The 4 (drug dose) $\times 10$ (seconds) mixed ANOVA con-

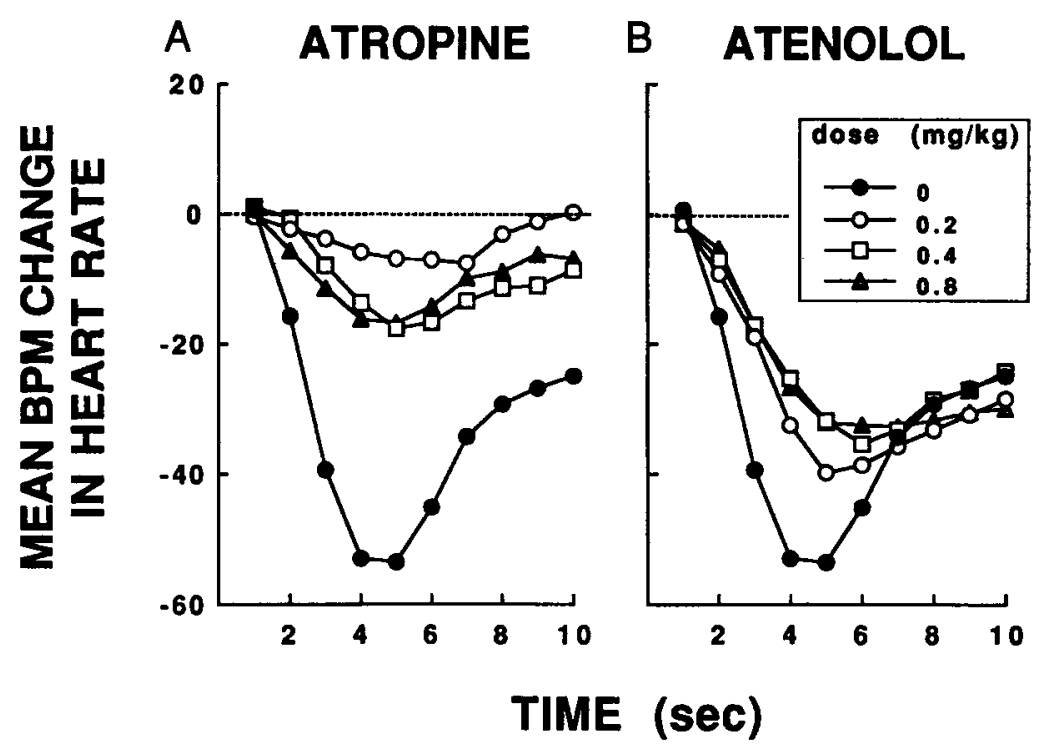

Figure 2. Mean beats-per-minute (BPM) change in heart rate observed after pretreatment with saline $(0 \mathrm{mg} / \mathrm{kg}), 0.2,0.4$, or $0.8 \mathrm{mg} / \mathrm{kg}$ atropine $(A)$, and atenolol (B) in Experiment 2. The data are collapsed across the first three presentations of the olfactory conditioned stimulus. 
ducted on the change in HR data for the saline and three atropine groups yielded significant main effects of drug dose $[F(3,36)=5.54, p<.01]$ and seconds $[F(9,324)=$ $18.49, p<.01]$, as well as a significant interaction between drug dose and seconds $[F(27,324)=3.63, p<$ $.01]$. Subsequent contrast analyses conducted on the data from each group to compare the change in HR observed during the CS against baseline indicated that the atropine groups did not differ significantly on any second, although the HR change did approach statistical significance on 3 of the $10 \mathrm{sec}$ for each group [largest $F(1,9)=3.02]$. The response of the saline group differed from baseline on Seconds 2-10 [smallest $F(1,9)=9.94$ ]

Atenolol. The 4 (drug dose) $\times 10$ (seconds) mixed ANOVA that was conducted to compare the saline and three atenolol groups yielded a significant main effect of seconds $[F(9,324)=53.65, p<.01]$ and a drug dose $\times$ seconds interaction $[F(27,324)=2.78, p<.05]$. Subsequent Newman-Keuls tests indicated that on Seconds 3 and 5 , the saline group evidenced larger bradycardia than the three atenolol groups $(p<.05)$, which did not differ from each other. Thus, atenolol had the effect of slightly attenuating the unconditioned HR response. These data are presented in Figure 2B, where the saline group has been reproduced.

Baseline HR. A one-way ANOVA was employed to compare the animals' baseline HRs, averaged over the three trials, across the seven drug dose groups. This analysis yielded a significant main effect of drug dose $[F(6,63)=25.51, p<.01]$. Post hoc comparisons revealed that the three atenolol groups had lower baseline HRs than the saline and atropine groups, which did not differ from each other. Mean ( $\pm S E M)$ baseline HRs for all seven groups are presented in Table 1.

\section{Discussion}

These results suggest that the olfactory stimulus simultaneously evoked activation of the PNS division and withdrawal of the SNS division (Berntson, Cacioppo, \& Quigley, 1991), with activation of the PNS predominating. This was evidenced by the capacity of both atropine and atenolol to attenuate the phasic cardiac response. This pattern of simultaneous changes in the two ANS divisions is not typical of orienting responses generally, which are more often reported to arise solely from activation of the PNS (Graham, 1979; Lacey \& Lacey, 1970; Saiers et al., 1989). Moreover, this pattern of reciprocal changes in the SNS and PNS is not typical of the 16-day- old rat, since the decrease in HR elicited by a novel auditory stimulus at this age is completely blocked by administration of atropine (Saiers et al., 1989). Further research on olfactory stimuli will be needed to determine the generality of this pattern of autonomic nervous sytem (ANS) responding.

\section{EXPERIMENT 3}

The purpose of Experiment 3 was to examine the autonomic division(s) responsible for the generation of the conditioned acceleratory response observed to the olfactory stimulus after pairings with shock. Accelerations in HR have historically been thought to reflect an increase in SNS activity (Cannon, 1929). Because acceleration of the heart presumably reflects a preparation for "fight or flight" responses, it follows that the enhanced metabolic demands placed upon an organism about to engage in such physical activities would evoke a preparatory increase in sympathetic outflow. Pharmacological analysis of the autonomic mechanisms mediating conditioned increases in HR, however, do not always support a simple model of SNS activation. Iwata and LeDoux (1988), for example, reported that the conditioned acceleratory response to a tone paired with shock in rats was the result of coactivation of the PNS and SNS divisions. Blocking the PNS resulted in a larger acceleratory response to the tone CS, whereas blocking the SNS unmasked a large deceleratory response. D. H. Cohen and Pitts (1968) and Gold and D. H. Cohen (1981) found that the conditioned tachycardia to a visual stimulus in the pigeon was mediated through an increase in SNS outflow accompanied by withdrawal of the PNS.

On the basis of this work with adult animals, it is believed that increases in HR are most likely to result from activation of the SNS (Cannon, 1929; Iwata \& LeDoux, 1988; Katcher et al., 1969; Kazis et al., 1973; Kurtz \& Campbell, in press), either exclusively or together with a concomitant change in PNS activity (Berntson et al., 1991; Quigley \& Berntson, 1990). However, in young rats, the cardioacceleration may result from withdrawal of vagal inputs (Haroutunian \& Campbell, 1982; Kurtz \& Campbell, in press).

\section{Method}

Subjects. The subjects were 40 experimentally naive 16 -day-old $( \pm 1)$ Sprague-Dawley rat pups from 13 litters, maintained as those described in Experiment 1. No more than 1 animal per litter served in a given experimental condition.

Table 1

Mean $( \pm S E M)^{*}$ Baseline Heart Rates of Animals in Experiment 2 Given Saline $(0 \mathrm{mg} / \mathrm{kg})$; $0.2,0.4$, or $0.8 \mathrm{mg} / \mathrm{kg}$ Atropine Methyl Nitrate; or $0.2,0.4$, or $0.8 \mathrm{mg} / \mathrm{kg}$ Atenolol

\begin{tabular}{|c|c|c|c|c|c|c|c|c|c|c|c|c|c|}
\hline \multirow{2}{*}{\multicolumn{2}{|c|}{0}} & \multicolumn{6}{|c|}{ Atropine } & \multicolumn{6}{|c|}{ Atenolol } \\
\hline & & \multicolumn{2}{|c|}{0.2} & \multicolumn{2}{|c|}{0.4} & \multicolumn{2}{|c|}{0.8} & \multicolumn{2}{|c|}{0.2} & \multicolumn{2}{|c|}{0.4} & \multicolumn{2}{|c|}{0.8} \\
\hline$M$ & $S E M$ & $M$ & $S E M$ & $M$ & $S E M$ & $M$ & $S E M$ & $M$ & $S E M$ & $M$ & $S E M$ & $M$ & $S E M$ \\
\hline 480.1 & 12.4 & 491.0 & 12.8 & 489.3 & 4.7 & 502.0 & 11.6 & 407.8 & 8.2 & 406.8 & 10.6 & 399.8 & 13.0 \\
\hline
\end{tabular}

Note-Data are collapsed across three trials. *Standard error of the mean. 
Procedure. The animals were divided into four groups ( $n s=$ 10/group). Three of these groups were given 15 odor-shock pairings; the fourth group was given explicitly unpaired presentations of the odor CS and shock US. Training for the paired and unpaired groups was the same as that described in Experiment 1.

Immediately following the final training trial, the paired animals were removed from the chamber, injected intraperitoneally with saline, $0.4 \mathrm{mg} / \mathrm{kg}$ atropine methyl nitrate, or $0.4 \mathrm{mg} / \mathrm{kg}$ atenolol, and returned to the chamber for an additional $15 \mathrm{~min}$. The unpaired animals were injected with saline after training. Next, a series of four extinction trials was given. The interstimulus interval between $\mathrm{CS}$ presentations during the test varied from 90 to $150 \mathrm{sec}$.

Heart rate during acquisition and testing was recorded during a 5 -sec prestimulus period and during the 10 -sec CS. Changes from baseline HR were calculated for each second of the CS on each trial. The aquisition data were collapsed across blocks of three trials, as in Experiment 1.

\section{Results}

Acquisition. All of the groups initially exhibited a dramatic deceleration in HR to the presentation of the olfactory stimulus. The 4 (group) $\times 10$ (seconds) mixed ANOVA conducted on the data averaged over the first three conditioning trials (Trial Block 1) yielded a significant main effect of seconds $[F(9,324)=34.68, p<$ $.01]$. The main effect of group and the group $\times$ seconds interaction were both nonsignificant. There were no differences between the four groups in the magnitude or form of this bradycardia.

Acquisition proceded exactly as that in Experiment 1 (see Figure 1). All three of the paired groups displayed a long-latency tachycardia as early as the second trial block, whereas the HR of the unpaired animals varied around baseline. Significant differences between the paired and unpaired groups were detected, beginning on Trial Block 2. This pattern held across Trial Blocks 3-5

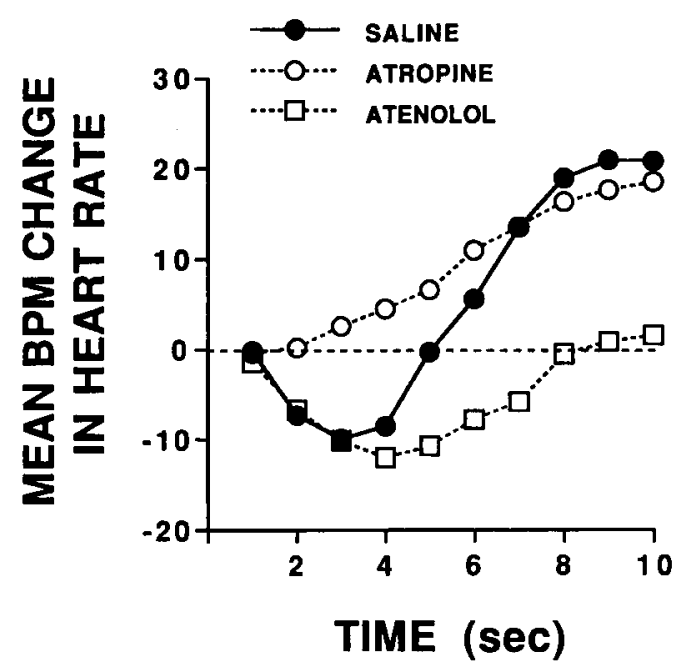

Figure 3. Mean beats-per-minute (BPM) change in heart rate over the 10-sec olfactory stimulus previously paired with transcutaneous shock. Data are collapsed across the four extinction trials given after acquisition training. Fifteen minutes prior to test, animals were injected with saline, $0.4 \mathrm{mg} / \mathrm{kg}$ atropine, or $0.4 \mathrm{mg} / \mathrm{kg}$ atenolol. [smallest $F(27,324)=3.48, p<.01]$. At no time during training did the three paired groups differ from one another, indicating that the groups were responding similarly prior to drug manipulation at test.

Testing: Autonomic mediation of the conditioned HR response. Preliminary analyses of the data obtained from the saline-treated paired animals indicated no significant extinction across the four test trials $(F<1)$. Therefore, the data from these trials were collapsed for analysis; they are presented in Figure 3 . The 4 (group) $\times$ 10 (seconds) mixed ANOVA conducted on the HR change scores yielded significant main effects of group $[F(3,36)=3.35, p<.05]$ and seconds $[F(9,324)=28.41$, $p<.01]$, as well as a significant group $\times$ seconds interaction $[F(27,324)=2.72, p<.05]$. As can be seen in the figure and confirmed statistically, the saline-treated paired animals showed a biphasic response to the olfactory CS. There was an initial cardiac deceleration-significantly different from baseline on Seconds 2 and 3followed by cardioacceleration that emerged by Second 7 [smallest $F(1,9)=5.52, p<.05$ ]. The responses of the unpaired group did not differ from baseline at any point, and therefore the data were not included in Figure 3.

Contrast analyses confirmed that atropine blocked the initial bradycardia to the odor CS, without affecting the final magnitude of the tachycardia attained. With parasympathetic blockade, the acceleratory response also emerged earlier than that with an intact PNS and was significantly elevated from baseline, beginning on Second 3 [smallest $F(1,9)=11.12, p<.01$ ]. In contrast, atenolol blocked the late-emerging acceleratory component of the conditioned response and resulted in a prolonged initial bradycardia. The bradycardia differed from baseline on Seconds 2-6 [smallest $F(1,9)=5.16$, $p<.05$ ], at which time HR returned to baseline levels. These results suggest that the early deceleratory component of the conditioned response resulted mainly from activation of the PNS, but the later acceleratory response was produced solely by activation of the SNS.

Baseline HR. A one-way ANOVA conducted on the prestimulus baseline HRs yielded a significant main effect of group $[F(3,36)=55.36, p<.01]$. The animals that had received atenolol had the lowest baseline HRs $(375.6 \pm 7.0 \mathrm{BPM})$, whereas those that had received atropine $(500.5 \pm 7.8 \mathrm{BPM})$ had basal HRs that were slightly higher than those of the saline-paired (466.5 $\pm 8.2 \mathrm{BPM}$ ) and unpaired (477.5 $\pm 8.7 \mathrm{BPM})$ groups. These latter two groups did not differ from each other.

\section{Discussion}

The conditioned cardiac response to an olfactory CS previously paired with shock was shown to be biphasic; each phase of the response was mediated through different autonomic control mechanisms. Following the acquisition phase and a 15 -min retention interval, the saline-treated paired animals displayed an initial bradycardia that was followed by an abrupt transition to tachycardia during the $10-\mathrm{sec}$ CS. Atropine simultane- 
ously blocked the initial deceleratory component of the conditioned response and permitted the occurrence of tachycardia earlier during the CS. Further, there was no effect of atropine on the magnitude of the conditioned increase in HR achieved by the end of the CS. In contrast, atenolol significantly prolonged the deceleratory component of the conditioned response and completely eliminated the terminal acceleratory phase. These results suggest that the initial cardiac deceleration resulted from PNS activation, and that the late acceleratory component was mediated solely by activation of the sympathetic division. There was evidence of coactivation of both ANS divisions during the middle of the $\mathrm{CS}$ period - the period of transition from bradycardia to tachycardia.

\section{GENERAL DISCUSSION}

The results of these experiments show that (1) the initial cardiac response to an unfamiliar olfactory stimulus is a decrease in HR driven by co-occurring increases in PNS activation and withdrawal of SNS inputs to the heart, and (2) the conditioned cardiac response to the same olfactory stimulus using a shock US is biphasic; the initial deceleratory response is driven primarily by activation of the PNS, and the subsequent acceleratory response is mediated by an increase in SNS stimulation of the heart.

These inferences concerning the sequential actions of the PNS and SNS evoked by unconditioned and conditioned olfactory stimuli are based entirely upon selective pharmacological blockade of the two divisions of the ANS independently. Pharmacological blockade studies, however, are subject to a variety of systematic biases that could alter interpretation of these data. Berntson and his colleagues have identified a number of potential biases, including (1) interactions of the two divisions of the ANS at the target organ, (2) reflexive or indirect alterations in the unblocked branch of the ANS, (3) incomplete receptor blockade, and (4) nonspecific effects of the blocking agents. Moreover, the preweanling rat may be particularly susceptible to systematic biases such as these because of the many changes in physiological regulation that occur during development, as described in the introduction. To address these issues, Berntson, $\mathrm{Ca}$ cioppo, and Quigley (in press) have proposed two inferential models that can be used to evaluate the validity of blockade studies: a subtractive model and a residual model. Each of these models yields an estimate of sympathetic and parasympathetic activity for each blockade condition. Thus, blockade of the sympathetic nervous system with atenolol yields a subtractive estimate of change in SNS activity ( $\left.\mathrm{s}^{\prime \prime}\right)$ and a residual estimate of PNS activity $\left(\mathrm{p}^{\prime}\right)$ elicited by any given test stimulus or experimental condition. For example, following administration of atenolol, a subtractive estimate of SNS influence can be obtained by determining the degree to which the cardiac response is attenuated relative to that of the saline control group, and a residual estimate of PNS activity can be obtained by determining the magnitude of the remaining response. The advantage of these separate estimates of SNS and PNS activity is that they can be used to provide a quantitative index of systematic bias. If systematic biases occur, the residual and subtractive estimates of PNS ( $\mathrm{p}^{\prime}$ and $\left.\mathrm{p}^{\prime \prime}\right)$ or SNS $\left(\mathrm{s}^{\prime}\right.$ and $\left.s^{\prime \prime}\right)$ activity will be highly divergent. Conversely, when systematic bias is low, these estimates should be similar.

To apply Berntson et al.'s (in press) model to the present results, changes in IBIs in milliseconds, instead of
A ORIENTING RESPONSE

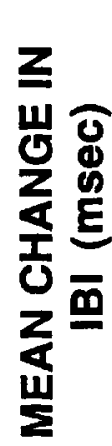

CONDITIONED RESPONSE

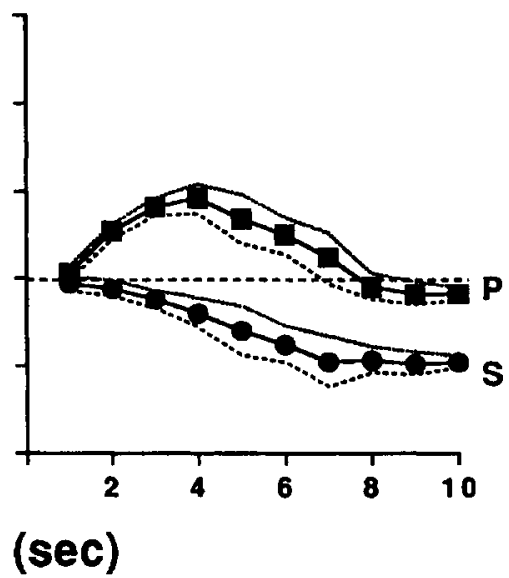

Figure 4. Graphic representations of the estimates of sympathetic (S) and parasympathetic (P) control derived from application of the model described by Berntson, Cacioppo, and Quigley (in press). The dashed lines represent the subtractive $\left(\mathrm{s}^{\prime \prime}\right.$ and $\left.\mathrm{p}^{\prime \prime}\right)$ estimates, and the dotted lines represent the residual $\left(s^{\prime}\right.$ and $p^{\prime}$ ) estimates of the contribution of the two ANS divisions to the unconditioned $(A)$ and conditioned (B) cardiac changes observed in Experiments 2 and 3, respectively. (IBI, interbeat interval.) 
changes in $\mathrm{HR}$, were used as the database to calculate the subtractive and residual estimates of the changes in sympathetic and parasympathetic activity evoked by the olfactory stimulus. Heart period has been identified as being more directly related to autonomic outflow than heart rate (see Berntson et al., 1991; Berntson, Quigley, Jang, \& Boysen, 1990).

Figure 4A shows the results of this analysis on the unconditioned cardiac response elicited by the olfactory stimulus in Experiment 2. The estimates were calculated from the data of the saline, $0.4 \mathrm{mg} / \mathrm{kg}$ atropine, and $0.4 \mathrm{mg} / \mathrm{kg}$ atenolol groups shown in Figure 2. The dashed lines in the figure represent the subtractive estimates ( $s^{\prime \prime}$ and $\left.p^{\prime \prime}\right)$, the dotted lines represent the residual estimates $\left(s^{\prime}\right.$ and $\left.p^{\prime}\right)$, and the solid lines depict the average of those two estimates. During the first $4 \mathrm{sec}$ of the olfactory stimulus, the residual estimates of PNS activity obtained from blockade of the sympathetic branch with atenolol are nearly identical to the subtractive estimates obtained through blockade of the PNS with atropine. The same pattern occurs for the subtractive and residual estimates of SNS activity. During the latter half of the stimulus presentation, the two estimates of SNS and PNS activity diverged as the cardiac response began to return to baseline. This pattern of increasing error is not surprising, since individual animals may habituate to the olfactory stimulus at different rates, thereby increasing the variability of the estimates of the autonomic mediation of the cardiac response. These data also clearly show the early SNS component in the production of the orienting response.

When the data of Experiment 3 were analyzed in the same fashion, the results were somewhat different. As can be seen in Figure 4B, the two estimates of PNS and SNS activity during conditioning are in extremely close agreement throughout the entire $10 \mathrm{sec}$ of the olfactory CS. This remarkable convergence of subtractive and residual estimates of ANS control of the heart greatly increases our confidence in the validity of our interpretation of these results. In particular, these analyses further confirm the following conclusions: (1) that the unconditioned decrease in HR evoked by a novel olfactory stimulus (orienting response) was mediated by activation of the PNS accompanied by a considerably smaller withdrawal of SNS activation of the heart, and (2) that the cardiac response to the olfactory stimulus following 15 conditioning trials is biphasic, and that the initial deceleratory response is driven primarily by activation of the PNS, and the subsequent acceleratory response is mediated by an increase in SNS stimulation of the heart.

The change from predominantly parasympathetic to sympathetic mediation of the HR response as a function of aversive training suggests a shift in the neural pathways subserving the unconditioned and conditioned cardiac responses (see Adams, 1979). Numerous researchers have reported that the amygdala, particularly the central nucleus, constitutes part of a major pathway for the expression of conditioned responses, including autonomic responses (e.g., Davis, 1992; Francis, Her- nandez, \& Powell, 1981; Iwata, LeDoux, \& Reis, 1986). The amygdala does not, however, seem to be involved in the production of behavioral or autonomic orienting responses to novel stimuli (Gallagher \& Holland, 1992; Kapp, Markgraf, Wilson, Pascoe, \& Supple, 1991; Sananes \& Campbell, 1989). Bilateral amygdaloid lesions, although effective in blocking the expression of a conditioned HR response, do not affect the orienting response to a novel stimulus (Sananes \& Campbell, 1989). The cerebellar vermis also has been implicated in the production of conditioned changes in HR, but not in the unconditioned $\mathrm{HR}$ response to a novel auditory stimulus (Supple \& Leaton, 1990a, 1990b). From these findings, it appears that one pathway is required for the generation of the unlearned cardiac deceleration elicited by the olfactory stimulus, but that after aversive Pavlovian training, another pathway, which promotes the conditioned cardiac response, is engaged. This latter pathway appears to involve the amygdala and its efferents, possibly one to the lateral hypothalamus (Iwata et al., 1986; LeDoux, Iwata, Cicchetti, \& Reis, 1988) or cerebellar vermis (Supple \& Leaton, 1990a, 1990b). An analysis of the neural pathways involved in the inception of these phasic cardiac responses to olfactory stimuli in the young rat, both learned and unlearned, could lead to further understanding of the neuroanatomical substrates involved in olfactory learning in general. How these pathways are altered or shifted during the process of acquisition and how they develop functionally during ontogeny (see Hunt, Richardson, \& Campbell, 1994; Sananes \& Campbell, 1989; Sananes et al., 1988) are issues of considerable importance.

Another interesting question is: Which of these presumably independent neural circuits (unconditioned or conditioned) is involved in the production of the early deceleratory component of the conditioned response (Experiment 3)? Is this deceleration truly part of the conditioned response to the olfactory stimulus, or is it in part some residual manifestation of the initial orienting response? Given that this stage of the response is not observed at the end of the training session (see Figure 1), but is seen after a short retention interval, suggests that it may reflect an attentional (orienting) process. However, the results of Experiment 3 indicate that the autonomic control over this deceleratory component differs from that controlling the orienting response (Experiment 2). The deceleratory orienting response results from an increase in PNS activation accompanied by a smaller withdrawal of SNS activity. In contrast, the early component of the conditioned response is subserved by activation of the PNS alone. Because the direction of the response and its autonomic origins collectively do not advance a clear interpretation, an analysis of the neural correlates of these responses could shed light on this question. For example, if the early deceleratory component does involve the circuitry involved in conditioned response generation, then amygdala lesions should abolish it. If, on the other hand, it is an indication of a residual orienting response, 
then such lesions should have no consequence on this phase of the cardiac response.

\section{REFERENCES}

ADAMS, D. B. (1979). Brain mechanisms for offense, defense, and submission. Behavioral \& Brain Sciences, 2, 201-241.

ADOLPH, E. F. (1965). Capacities for regulation of heart rate in fetal, infant, and adult rats. Americal Journal of Physiology, 209, 10951105.

ADOLPH, E. F. (1968). Origins of physiological regulations. New York: Academic Press.

Berntson, G. G., Caciopro, J. T., \& Quigley, K. S. (1991). Autonomic determinism: The modes of autonomic control, the doctrine of autonomic space, and the laws of autonomic constraint. Psychological Review, 98, 459-487.

Berntson, G. G., CACIOPPO, J. T., \& Quigley, K. S. (in press). Autonomic cardiac control: I. Estimation and validation from pharmacological blockades. Psychophysiology.

Berntson, G. G., Quigley, K. S., JANG, J., \& Boysen, S. T. (1990). A conceptual approach to artifact identification: Application to heart period data. Psychophysiology, 27, 568-598.

Campbell, B. A., Hayne, H., \& Richardson, R. (Eds.) (1992). Attention and information processing in infants and adults. Hillsdale, NJ: Erlbaum.

CANNON, W. B. (1929). Bodily changes in pain, hunger, fear, and rage (2nd ed.). New York: Appleton.

Conen, D. H., \& Pitrs, L. H. (1968). Vagal and sympathetic components of conditioned cardioacceleration in the pigeon. Brain Research, 9, 15-31.

CoHEN, H. L. (1986). Development of autonomic innervation in mammalian myocardium. In P. M. Gootman (Ed.), Developmental neurobiology of the autonomic nervous system (pp. 159-191). Clifton, NJ: Humana.

Coles, M. G. H., Jennings, J. R., \& Stern, J. A. (Eds.) (1984). Psychophysiology: A festschrift for John and Beatiruce Lacey. New York: Van Nostrand Reinhold.

DAvis, M. (1992). The role of the amygdala in fear and anxiety. $A n$ nual Review of Neuroscience, 15, 353-375.

DYKMAN, R. A., \& GANTT, W. H. (1959). The parasympathetic component of unlearned and acquired cardiac responses. Journal of Comparative \& Physiological Psychology, 52, 163-167.

FANSELOW, M. S. (1991). The midbrain periacqueductal gray as a coordinator of action in response to fear and anxiety. In A. Depaulis \& R. Bandler (Eds.), The midbrain periacqueductal gray matter: Functional, anatomical, and neurochemical organization (pp. 151173). New York: Plenum.

Francis, J., Hernandez, L. L., \& Powell, D. A. (1981). Lateral hypothalamic lesions: Effects on Pavlovian cardiac and eyeblink conditioning in the rabbit. Brain Research Bulletin, 6, 155-163.

Gallagher, M., \& Holland, P. C. (1992). Understanding the function of the central nucleus: Is simple conditioning enough? In J. P. Aggleton (Ed.), The amygdala: Neurobiological aspects of emotion, memory, and mental dysfunction (pp. 307-321). New York: WileyLiss.

Gold, M. R., \& CoHen, D. H. (1981). Modification of the discharge of vagal neurons during learned heart rate change. Science, 214, 345-347.

GrahaM, F. K. (1979). Distinguishing among orienting, defense, and startle reflexes. In H. D. Kimmel, E. H. van Olst, \& J. F. Orlebeke (Eds.), The orienting reflex in humans (pp. 137-167). Hillsdale, NJ: Erlbaum.

Graham, F. K., \& Clifton, R. K. (1966). Heart-rate change as a component of the orienting response. Psychological Bulletin, 65, 305320.

Haroutunian, V., \& Campbell, B. A. (1982). Neural control of the heart-rate orienting response in preweanling rats. Behavioral \& Neural Biology, 36, 24-39.

HOFER, M. A. (1974). The role of early experience in the development of autonomic regulation. In L.V. DiCara (Ed.), Limbic and autonomic nervous system research (pp. 195-221). New York: Plenum.
HOFER, M. A., \& REISER, M. F. (1969). The development of cardiac rate regulation in preweanling rats. Psychosomatic Medicine, 31, 372388.

Hunt, P. S., Richardson, R., \& CAmpbell, B. A. (1994). Delayed development of fear-potentiated startle in rats. Behavioral Neuroscience, 108, 1-12.

IwATA, J., \& LeDoux, J. E. (1988). Dissociation of associative and nonassociative concomitants of classical fear conditioning in the freely behaving rat. Behavioral Neuroscience, 102, 66-76.

IWATA, J., LeDOuX, J. E., \& ReIS, D. J. (1986). Destruction of intrinsic neurons in the lateral hypothalamus disrupts the classical conditioning of autonomic but not behavioral emotional responses in the rat. Brain Research, 368, 161-166.

Kapp, B. S., Markgraf, C. G., Wilson, A., Pascoe, J. P., \& Supple, W. F. (1991). Contribution of the amygdala and anatomically related structures to the acquisition and expression of aversively conditioned responses. In L. Dachowski \& C. F. Flaherty (Eds.), Current topics in animal learning: Brain, emotion, and cognition (pp. 311346). Hillsdale, NJ: Erlbaum.

Katcher, A. H., Solomon, R. L., TURner, L. H., LoLordo, V., OVerMIER, J. B., \& Rescorla, R. A. (1969). Heart rate and blood pressure responses to signalled and unsignalled shocks: Effects of cardiac sympathectomy. Journal of Comparative \& Physiological Psychology, 68, 163-174.

Kazis, E., Milligan, W. L., \& Powell, D. A. (1973). Autonomicsomatic relationships: Blockade of heart rate and corneo-retinal potential responses. Journal of Comparative \& Physiological Psychology, 84, 98-110.

KEPPEL, G. (1982). Design and analysis: A researcher's handbook. Englewood Cliffs, NJ: Prentice-Hall.

Kimmel, H. D., van Olst, E. H., \& OrLebeke, J. F. (EDs.) (1979). The orienting reflex in humans. Hillsdale, NJ: Erlbaum.

Kurtz, M. M., \& CAMPBell, B. A. (in press). Paradoxical autonomic responses to aversive stimuli in the developing rat. Behavioral Neuroscience.

LACEY, J. I., \& LACEY, B. C. (1970). Some autonomic-central nervous system interrelationships. In P. Black (Ed.), Physiological correlates of emotion (pp. 205-227). New York: Academic Press.

LARSON, S. K., \& PORGES, S. W. (1982). The ontogeny of heart period patterning in the rat. Developmental Psychobiology, 15, 519-528.

LeDouX, J. E., Iwata, J., Cicchetti, P., \& Reis, D. J. (1988). Different projections of the central amygdaloid nucleus mediate autonomic and behavioral correlates of conditioned fear. Journal of Neuroscience, 8, 2517-2529.

OBRIST, P. A. (1968). Heart rate and somatic-motor coupling during classical aversive conditioning in humans. Journal of Experimental Psychology, 77, 180-193.

Obrist, P. A., Wood, D. M., \& Perez-Reyes, M. (1965). Heart rate during conditioning in humans: Effects of UCS intensity, vagal blockade, and adrenergic block of vasomotor activity. Journal of Experimental Psychology, 70, 32-42.

Pappano, A. J. (1977). Ontogenetic development of autonomic neuroeffector transmission and transmitter reactivity in embryonic and fetal hearts. Pharmacological Reviews, 29, 3-33.

QUIGLEY, K. S., \& BERNTSON, G. G. (1990). Autonomic origins of cardiac responses to nonsignal stimuli in the rat. Behavioral Neuroscience, 104, 751-762.

Richardson, R., Hess, M. F., \& Campbell, B. A. (1994). The orienting response to brief auditory stimuli in preweanling and adult rats. Developmental Psychobiology, 27, 93-100.

Richardson, R., Siegel, M., \& CAMPBell, B. A. (1988). Unfamiliar environments impair information processing as measured by behavioral and cardiac orienting responses to auditory stimuli in preweanling and adult rats. Developmental Psychobiology, 21, 491503.

Richardson, R., Wang, P., \& Campbell, B. A. (1994). Developmental and pharmacological analysis of the cardiac response to an acoustic startle stimulus. Manuscript submitted for publication.

Saiers, J. A., Richardson, R., \& Campbell, B. A. (1989). Pharmacological dissociation of heart rate and somatomotor components of the orienting response. Psychobiology, 17, 418-423.

Sananes, C. B., \& Campbell, B. A. (1989). Role of the central nu- 
cleus of the amygdala in olfactory heart rate conditioning. Behavioral Neuroscience, 103, 519-525.

Sananes, C. B., Gaddy, J. R., \& Campbell, B. A. (1988). Ontogeny of conditioned heart rate to an olfactory stimulus. Developmental Psychobiology, 21, 117-133.

SCHNEIRLA, T. C. (1959). An evolutionary and developmental theory of biphasic processes underlying approach and withdrawal. In M. R. Jones (Ed.), The Nebraska Symposium on Motivation (pp. 1-41). Lincoln: University of Nebraska Press.

SCHNEIRLA, T. C. (1965). Aspects of stimulation and organization in approach/withdrawal processes underlying vertebrate behavioral development. In D. S. Lehrman, R. A. Hinde, \& E. Shaw (Eds.), $A d$ vances in the study of behavior (Vol. 1, pp. 1-74). New York: Academic Press.

SLOTKIN, T. A. (1986). Endocrine control of synaptic development in the sympathetic nervous system: The cardiac-sympathetic axis. In P. M. Gootman (Ed.), Developmental neurobiology of the autonomic nervous system (pp. 97-133). Clifton, NJ: Humana.
SupPle, W. F., \& Leaton, R. N. (1990a). Cerebellar vermis: Essential for classically conditioned bradycardia in the rat. Brain Research, 509, 17-23

SUPPLE, W. F., \& Leaton, R. N. (1990b). Lesions of the cerebellar vermis and cerebellar hemispheres: Effects on heart rate conditioning in rats. Behavioral Neuroscience, 104, 934-947.

TUCKER, D. C. (1985). Components of functional sympathetic control of heart rate in neonatal rats. American Journal of Physiology, 248, R601-R610.

TuCKER, D. C., \& Domino, J. V. (1988). Balance among autonomic controls of heart rate in neonatal spontaneously hypertensive and borderline hypertensive rats. Journal of the Autonomic Nervous System, 22, 11-21.

(Manuscript received March 14, 1994; revision accepted for publication May 25, 1994.) 